\title{
Silica-Bonded $N$-Propyl Diethylenetriamine Sulfamic Acid as a Recyclable Solid Acid Catalyst for the Synthesis of $\alpha$-Aminonitriles
}

\author{
Tahere RAHI ${ }^{1}$, Mojtaba BAGHERNEJAD ${ }^{1}$, Khodabakhsh NIKNAM ${ }^{2, *}$ \\ ${ }^{1}$ Department of Chemistry, Gachsaran Branch, Islamic Azad University, Gachsaran, Iran \\ ${ }^{2}$ Department of Chemistry, Faculty of Sciences, Persian Gulf University, Bushehr 75169, Iran
}

\begin{abstract}
Silica-bonded $N$-propyl diethylenetriamine sulfamic acid (SBPDSA) is employed as a recyclable catalyst to synthesize $\alpha$-aminonitriles. These syntheses involved one-pot condensation of an aldehyde, an amine, and trimethylsilyl cyanide under mild reaction conditions at room temperature. SBPDSA was recycled seven times in the condensation of benzaldehyde, aniline, and trimethylsilyl cyanide without reduction of its catalytic activity.
\end{abstract}

Key words: silica-bonded $N$-propyl diethylenetriamine sulfamic acid; $\alpha$-aminonitrile; solid acid; aldehyde; heterogeneous catalyst

CLC number: O643 Document code: A

Received 15 January 2012. Accepted 23 February 2012.

*Corresponding author.Tel: +98-771-4541494; Fax: +98-771-4545188; E-mail: niknam@pgu.ac.ir, khniknam@gmail.com

This work was supported by Islamic Azad University.

English edition available online at Elsevier ScienceDirect (http://www.sciencedirect.com/science/journal/18722067).

The addition of cyanide anions to imines (the Strecker reaction) [1] is one of the most important and straightforward method to synthesize $\alpha$-aminonitriles, which are useful intermediates for the synthesis of amino acids [2] and nitrogen-containing heterocycles such as thiadiazoles and imidazoles $[3,4]$. The classical Strecker reaction is usually carried out in aqueous solution and the work-up procedure is tedious. Thus, several modifications of the Strecker reaction have been developed using a variety of cyanide reagents [5], such as diethyl phosphorocyanidate and $\alpha$-trimethylsiloxynitriles, as well as catalysts including $\mathrm{InCl}_{3}$ [6], [bmim] $\mathrm{BF}_{4}$ [7], montmorillonite KSF clay [8], silica sulfuric acid [9], $\mathrm{I}_{2}[10], \mathrm{Fe}(\mathrm{Cp})_{2} \mathrm{PF}_{6}$ [11], xanthan sulfuric acid [12], hydrophobic sulfonic acid-based nanoreactors [13], silica-bonded S-sulfonic acid [14], sulfamic acid-functionalized magnetic $\mathrm{Fe}_{3} \mathrm{O}_{4}$ nanoparticles [15], and silica-based ionic liquids [16], under various reaction conditions. Trimethylsilyl cyanide (TMSCN) is a safe and effective source of cyanide anions for the nucleophilic addition reactions of imines under mild conditions [17,18]. However, many of these methods require the use of expensive reagents, harsh conditions, extended reaction times, and also require tedious work-up that generates a large amount of toxic waste. Furthermore, many of these catalysts are deactivated or sometimes decomposed by amines and water that are present during imine formation. To overcome these problems, one-pot procedures have recently been developed for this transformation [19].

Several types of solid sulfonic acid-functionalized silica (both amorphous and ordered) have been synthesized and used as an alternative to traditional sulfonic acid resins and homogeneous acids to catalyze chemical transformations [20-29]. In continuation of our studies on the design and application of solid acid catalysts in organic transformations [21-29], herein, we describe the application of silica-bonded $\mathrm{N}$-propyl diethylenetriamine sulfamic acid (SBPDSA) as recyclable catalyst for the synthesis of $\alpha$-aminonitriles.

\section{Experimental}

\subsection{Catalyst preparation}

\subsubsection{Preparation of silica propyl chloride}

Silica was first immersed in hydrochloric acid for $24 \mathrm{~h}$ and then washed with deionized water and dried under vacuum at $120{ }^{\circ} \mathrm{C}$ for $8 \mathrm{~h}$. The activated silica $(25.0 \mathrm{~g})$ was suspended in dry toluene $(300 \mathrm{ml})$ and then an excess of 3-chloropropyltrimethoxysilane $(25.0 \mathrm{ml})$ was added followed by triethylamine $(2.5 \mathrm{ml})$ as a catalyst. The suspension was mechanically stirred as it was heated under reflux for $48 \mathrm{~h}$. The reaction was cooled to room temperature, filtered, and then washed sequentially with toluene, etha- 
nol-water, deionized water, and methanol. The produced was dried under vacuum at $60{ }^{\circ} \mathrm{C}$ for $4 \mathrm{~h}$ to give chloropropyl silica (28.45 g).

\subsubsection{Preparation of 3-diethylenetriamine-propyl silica (DTPS)}

To a mixture of chloropropyl silica (25 g) in anhydrous xylene $(250 \mathrm{ml})$, an excess of diethylenetriamine $(25 \mathrm{ml})$ was added and the mixture was heated under reflux with stirring for $24 \mathrm{~h}$. The reaction was cooled to room temperature, filtered, and then washed with xylene and a large excess of ethanol. The product was dried under vacuum overnight at $80^{\circ} \mathrm{C}$ to give DTPS (26.23 g, Scheme 1).

\subsubsection{Preparation of SBPDSA}

To a magnetically stirred mixture of DTPS $(5 \mathrm{~g})$ in $\mathrm{CHCl}_{3}$ $(20 \mathrm{ml})$ at $0{ }^{\circ} \mathrm{C}$, chlorosulfonic acid $(2.00 \mathrm{~g}, 18 \mathrm{mmol})$ was added dropwise over $2 \mathrm{~h}$. After the addition was complete, the mixture was stirred for $3 \mathrm{~h}$ until all $\mathrm{HCl}$ was removed from the reaction vessel. The mixture was filtered, washed with methanol $(30 \mathrm{ml})$, and then dried at room temperature to give SBPDSA as a white powder (5.39 g) [30].

\subsection{General procedure for the synthesis of $\alpha$-aminonitrile derivatives}

A mixture of aldehyde (1 mmol), amine $(1.5 \mathrm{mmol})$, TMSCN (1.5 mmol), and SBPDSA (0.07 g, $6.9 \mathrm{~mol} \%$ ) in EtOH $(2 \mathrm{ml})$ was stirred at room temperature for an appropriate time. The reaction progress was followed by TLC using silica gel plates (SILG/UV 254). After the reaction was complete, the reaction mixture was filtered and washed with warm ethanol $(5 \mathrm{ml} \times 3)$. After cooling, the corresponding $\alpha$-aminonitrile products were purified by recrystallization from hot ethanol. The recovered catalyst was dried and reused for subsequent runs.

Infrared (IR) spectra were measured on an IR spectrometer (Shimadzu, FT-IR-8000, Japan). ${ }^{1} \mathrm{H}$ and ${ }^{13} \mathrm{C}$ NMR spec- tra were obtained on Bruker Avance (DRX $500 \mathrm{MHz}$ and $400 \mathrm{MHz}$ ) instruments in $\mathrm{CDCl}_{3}$. Melting points were recorded on a melting point apparatus (SMP1) in open capillary tubes and are uncorrected. All products were characterized by comparison of their IR, ${ }^{1} \mathrm{H}$ and ${ }^{13} \mathrm{C}$ NMR spectroscopic data and their melting points with reported values [5-16].

Phenyl-phenylamino-acetonitrile (1a): white solid; recrystallized from ethanol; m.p. $80-82{ }^{\circ} \mathrm{C}\left(81-83{ }^{\circ} \mathrm{C}\right.$ [12]). IR (KBr, cm ${ }^{-1}$ ): v 3369 (s), 2236 (m), 1606 (s), 1498 (vs), 1240 (s), 1120 (s), 805 (s), 680 (s). ${ }^{1} \mathrm{H}$ NMR (400 MHz, $\left.\mathrm{CDCl}_{3}\right): \delta 4.04$ (brs, $\left.1 \mathrm{H}\right), 5.46(\mathrm{~s}, 1 \mathrm{H}), 6.81\left(\mathrm{dd}, 2 \mathrm{H}, J_{1}=8.5\right.$ $\left.\mathrm{Hz}, J_{2}=1.0 \mathrm{~Hz}\right), 6.93(\mathrm{t}, 1 \mathrm{H}, J=7.35 \mathrm{~Hz}), 7.28-7.33(\mathrm{~m}$, 2H), 7.47-7.51 (m, 3H), 7.62-7.65 (m, 2H). ${ }^{13} \mathrm{C}$ NMR (100 $\left.\mathrm{MHz}, \mathrm{CDCl}_{3}\right): \delta 50.29,114.2,118.2,120.36,127.29$, $129.38,129.61,131.4,133.99$, 144.7. Anal. Calcd for $\mathrm{C}_{14} \mathrm{H}_{12} \mathrm{~N}_{2}$ : C, 80.74; H, 5.81; N, 13.45; Found: C, 80.60; H, 5.62; N, 13.38 .

(4-Chloro-phenyl)-phenylamino-acetonitrile (1b): white solid; recrystallized from ethanol; m.p. 109-111 ${ }^{\circ} \mathrm{C}$ (111-112 ${ }^{\circ} \mathrm{C}$ [8]). IR (KBr, cm ${ }^{-1}$ ): v 3300 (s), 2950 (s), 2232 (m), 1598 (s), 1500 (vs), 1218 (s), 1158 (s), 935 (s), 890 (s), 830 (s), 760 (s), 690 (s). ${ }^{1} \mathrm{H}$ NMR (400 MHz, $\left.\mathrm{CDCl}_{3}\right): \delta$ $4.08(\mathrm{~d}, 1 \mathrm{H}, J=8.6 \mathrm{~Hz}), 5.45(\mathrm{~d}, 1 \mathrm{H}, J=8.6 \mathrm{~Hz}), 6.79(\mathrm{~d}$, $2 \mathrm{H}, J=7.8 \mathrm{~Hz}), 6.95(\mathrm{t}, 1 \mathrm{H}, J=7.4 \mathrm{~Hz}), 7.30(\mathrm{t}, 2 \mathrm{H}, J=8.9$ $\mathrm{Hz}), 7.46$ (d, 2H, $J=8.6 \mathrm{~Hz}), 7.57$ (d, $2 \mathrm{H}, J=8.4 \mathrm{~Hz}) .{ }^{13} \mathrm{C}$ NMR (100 MHz, $\left.\mathrm{CDCl}_{3}\right): \delta 49.68,114.34,117.88,120.61$, $128.65,129.57,129.66,132.44,135.64,144.39$.

(4-Bromo-phenyl)-phenylamino-acetonitrile (1c): white solid; recrystallized from ethanol; m.p. 101-103 ${ }^{\circ} \mathrm{C}$ (99-100 $\left.{ }^{\circ} \mathrm{C}[5]\right)$. IR (KBr, cm ${ }^{-1}$ ): $v 3300$ (s), 2960 (s), 2235 (m), 1598 (s), 1498 (vs), 1218 (s), 1158 (s), 930 (s), 890 (s), 825 (s), 760 (s), 690 (s). ${ }^{1} \mathrm{H}$ NMR (400 MHz, $\left.\mathrm{CDCl}_{3}\right): \delta 3.97(\mathrm{~d}, 1 \mathrm{H}$, $J=8.6 \mathrm{~Hz}), 5.32(\mathrm{~d}, 1 \mathrm{H}, J=8.6 \mathrm{~Hz}), 6.68(\mathrm{~d}, 2 \mathrm{H}, J=8.3$ $\mathrm{Hz}), 6.84$ (t, 1H, $J=7.4 \mathrm{~Hz}), 7.19$ (t, 2H, $J=8.1 \mathrm{~Hz}), 7.40$ $(\mathrm{d}, 2 \mathrm{H}, J=8.8 \mathrm{~Hz}), 7.49-7.52(\mathrm{~m}, 2 \mathrm{H}) .{ }^{13} \mathrm{C}$ NMR $(100$ $\left.\mathrm{MHz}, \mathrm{CDCl}_{3}\right): \delta 49.74,114.34,117.81,120.63,123.79$, 128.91, 129.66, 132.54, 132.97, 144.38 .

(2,4-Dichloro-phenyl)-phenylamino-acetonitrile (1d): white solid; recrystallized from ethanol; m.p. $115-117{ }^{\circ} \mathrm{C}$

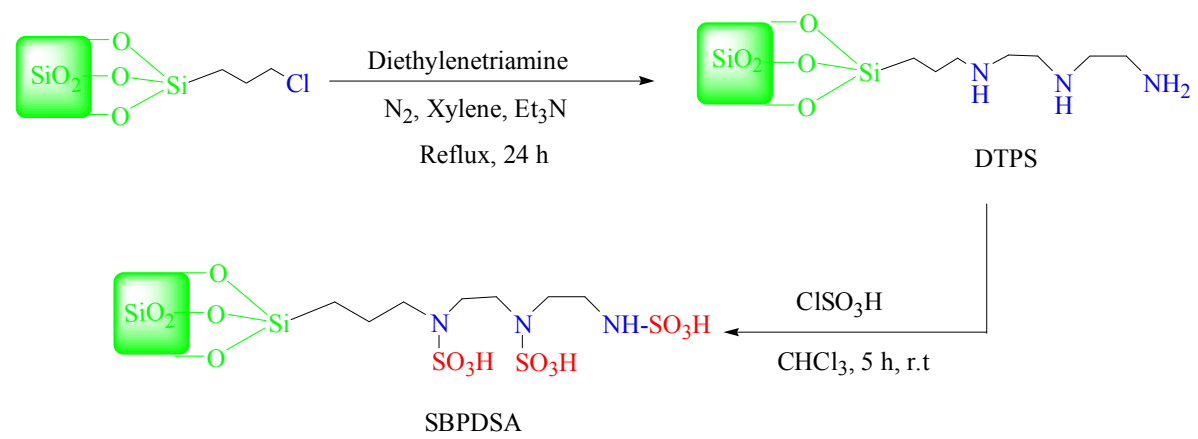

Scheme 1. Preparation of silica-bonded $N$-propyl diethylenetriamine sulfamic acid (SBPDSA). 
(115-117 ${ }^{\circ} \mathrm{C}$ [14]). IR ( $\left.\mathrm{KBr}, \mathrm{cm}^{-1}\right): v 3330$ (s), 2920 (s), 2230 (m), 1595 (s), 1550 (vs), 1380 (s), 1090 (s), 870 (s), 730 (s), 690 (s). ${ }^{1} \mathrm{H}$ NMR (300 $\mathrm{MHz}, \mathrm{CDCl}_{3}$ ): $\delta 4.05$ (brs, $1 \mathrm{H}), 5.70(\mathrm{~s}, 1 \mathrm{H}), 6.79\left(\mathrm{dd}, 2 \mathrm{H}, J_{1}=8.57 \mathrm{~Hz}, J_{2}=0.75 \mathrm{~Hz}\right)$, $6.94(\mathrm{t}, 1 \mathrm{H}, J=7.35 \mathrm{~Hz}), 7.27-7.32(\mathrm{~m}, 2 \mathrm{H}), 7.39$ (dd, 1H, $\left.J_{1}=8.28 \mathrm{~Hz}, J_{2}=2.07 \mathrm{~Hz}\right), 7.53(\mathrm{~d}, 1 \mathrm{H}, J=2.07 \mathrm{~Hz}), 7.70$ $(\mathrm{d}, 1 \mathrm{H}, J=8.48 \mathrm{~Hz}) .{ }^{13} \mathrm{C}$ NMR $\left(75 \mathrm{MHz}, \mathrm{CDCl}_{3}\right): \delta 47.69$, $114.41,117.39,120.76,128.14,129.66,129.89,130.42$, 134.35, 136.54, 144.32. Anal. Calcd for $\mathrm{C}_{14} \mathrm{H}_{10} \mathrm{Cl}_{2} \mathrm{~N}_{2}$ : C, 60.67; H, 3.64; N, 10.11; Found: C, 60.47; H, 3.37; N, 9.93.

(4-Methoxy-phenyl)-phenylamino-acetonitrile (1e): white solid; recrystallized from ethanol; m.p. $95-97{ }^{\circ} \mathrm{C}\left(94-95{ }^{\circ} \mathrm{C}\right.$ [8]). IR (KBr, cm ${ }^{-1}$ ): v 3340 (s), 2920 (s), 2235 (m), 1590 (s), 1500 (vs), 1300 (s), 1240 (s), 1180 (s), 1020 (s), 822 (s), 745 (s), 690 (s). ${ }^{1} \mathrm{H}$ NMR (300 MHz, $\left.\mathrm{CDCl}_{3}\right): \delta 3.86$ (s, $3 \mathrm{H}), 4.01$ (brs, 1H), 5.38 (s, 1H), 6.80 (d, 2H, $J=7.5 \mathrm{~Hz})$, 6.92 (t, 1H, $J=7.4 \mathrm{~Hz}), 6.99$ (d, 2H, $J=8.7 \mathrm{~Hz}), 7.29$ (dd, $\left.2 \mathrm{H}, J_{1}=7.2 \mathrm{~Hz}, J_{2}=1.2 \mathrm{~Hz}\right), 7.53(\mathrm{~d}, 2 \mathrm{H}, J=8.5 \mathrm{~Hz}) .{ }^{13} \mathrm{C}$ NMR (75 MHz, $\left.\mathrm{CDCl}_{3}\right): \delta 49.71,55.46,114.15,114.68$, $118.45,120.22$, 126, 128.65, 129.58, 144.77, 160.48. Anal. Calcd for $\mathrm{C}_{15} \mathrm{H}_{14} \mathrm{~N}_{2} \mathrm{O}$ : C, 75.61; H, 5.92; N, 11.76; Found: C, 75.41; H, 5.84; N, 11.60 .

(3,4,5-Trimethoxy-phenyl)-phenylamino-acetonitrile (1f): white solid; recrystallized from ethanol; m.p. $147-149{ }^{\circ} \mathrm{C}$ (147-149 ${ }^{\circ} \mathrm{C}$ [14]). IR ( $\mathrm{KBr}, \mathrm{cm}^{-1}$ ): v 3340 (s), 2950 (s), 2228 (s), 1595 (s), 1498 (vs), 1460 (s), 1422 (s), 1230 (s), 1125 (s), 1100 (s), 820 (s), 760 (s), 690 (s). ${ }^{1} \mathrm{H}$ NMR (300 $\left.\mathrm{MHz}, \mathrm{CDCl}_{3}\right): \delta 3.89$ (s, 3H), 3.91 (s, 6H), 4.1 (brs, 1H), $5.39(\mathrm{~s}, 1 \mathrm{H}), 6.80-6.83(\mathrm{~m}, 4 \mathrm{H}), 6.94(\mathrm{t}, 1 \mathrm{H}, J=7.4 \mathrm{~Hz})$, 7.28-7.34 (m, 2H). ${ }^{13} \mathrm{C}$ NMR $\left(75 \mathrm{MHz}, \mathrm{CDCl}_{3}\right): \delta$ 50.51, $56.35,60.93,104.36,114.20,118.20,120.43,129.34$, 129.63, 144.67, 153.87. Anal. Calcd for $\mathrm{C}_{17} \mathrm{H}_{18} \mathrm{~N}_{2} \mathrm{O}_{3}: \mathrm{C}$, 68.44; H, 6.08; N, 9.09; Found: C, 68.35; H, 5.98; N, 9.0.

(4-Chloro-phenylamino)-(3,4,5-trimethoxy-phenyl)-aceto nitrile (1g): white solid; recrystallized from ethanol; m.p. 145-148 ${ }^{\circ} \mathrm{C}$. IR (KBr, cm ${ }^{-1}$ ): v 3380 (vs), 2950 (m), 2910 (s), 2807 (m), 2340 (m), 1590 (vs), 1500 (vs), 1458 (s), 1418 (s), 1338 (s), 1310 (s), 1279 (s), 1240 (s), 1230 (vs), 1180 (m), 1081 (m), 999 (s), 810 (s), 720 (s). ${ }^{1} \mathrm{H}$ NMR (400 $\left.\mathrm{MHz}, \mathrm{CDCl}_{3}\right): \delta 3.87(\mathrm{~s}, 3 \mathrm{H}), 3.88(\mathrm{~s}, 6 \mathrm{H}), 4.12(\mathrm{~d}, 1 \mathrm{H}, J=$ $8.4 \mathrm{~Hz}), 5.32(\mathrm{~d}, 1 \mathrm{H}, J=8.0 \mathrm{~Hz}), 6.72(\mathrm{~d}, 2 \mathrm{H}, J=8.8 \mathrm{~Hz})$, $6.78(\mathrm{~s}, 2 \mathrm{H}), 7.23-7.26(\mathrm{~m}, 2 \mathrm{H}) .{ }^{13} \mathrm{C}$ NMR (100 MHz, $\left.\mathrm{CDCl}_{3}\right): \delta$ 50.42, 56.23, 60.89, 104.22, 115.35, 117.97, 125.05, 128.94, 129.45, 138.64, 143.33, 153.77. Anal. Calcd for $\mathrm{C}_{17} \mathrm{H}_{17} \mathrm{ClN}_{2} \mathrm{O}_{3}$ : C, 61.36; H, 5.15; N, 8.42; Found: $\mathrm{C}$, 61.13; H, 5.12; N, 8.28.

(3-Nitro-phenyl)-phenylamino-acetonitrile (1h): pale yellow solid; recrystallized from ethanol; mp 88-91 ${ }^{\circ} \mathrm{C}$ (89-92 ${ }^{\circ} \mathrm{C}$ [14]). IR (KBr, cm $\left.{ }^{-1}\right): v 3355$ (s), 2870 (s), 2240 (m), 1598 (s), 1530 (vs), 1342 (s), 1092 (s), 902 (s), 785 (s), 744 (s), 690 (s). ${ }^{1} \mathrm{H}$ NMR (300 MHz, $\mathrm{CDCl}_{3}$ ): $\delta 4.19$ (brs, $1 \mathrm{H}), 5.60(\mathrm{~s}, 1 \mathrm{H}), 6.80(\mathrm{~d}, 2 \mathrm{H}, J=8.1 \mathrm{~Hz}), 6.97$ (t, $1 \mathrm{H}, J=$
$7.7 \mathrm{~Hz}), 7.30(\mathrm{~m}, 2 \mathrm{H}), 7.70(\mathrm{t}, 1 \mathrm{H}, J=7.7 \mathrm{~Hz}), 8.01(\mathrm{~d}, 1 \mathrm{H}$, $J=8.1 \mathrm{~Hz}), 8.33(\mathrm{~d}, 1 \mathrm{H}, J=8.7 \mathrm{~Hz}), 8.53(\mathrm{~s}, 1 \mathrm{H}) .{ }^{13} \mathrm{C} \mathrm{NMR}$ $\left(75 \mathrm{MHz}, \mathrm{CDCl}_{3}\right): \delta 49.72,114.66,121.17,122.40,124.54$, 129.75, 130.51, 133.04, 143.94. Anal. Calcd for $\mathrm{C}_{14} \mathrm{H}_{13} \mathrm{~N}_{3} \mathrm{O}_{2}$ : C, 66.40; H, 4.38; N, 16.59; Found: C, 66.22; $\mathrm{H}, 4.22$; N, 16.38 .

Phenylamino-thiophen-2-yl)-acetonitrile (1i): white cream solid; recrystallized from ethanol; m.p. 97-99 ${ }^{\circ} \mathrm{C}$ (98-100 ${ }^{\circ} \mathrm{C}$ [11]). IR (KBr, cm $\left.{ }^{-1}\right): v 3357$ (s), 3100 (m), 2237 (m), 1599 (s), 1500 (vs), 1436 (s), 1348 (s), 1252 (s), 1149 (s), 1063 (s), 884 (s), 833 (s), 751 (s). ${ }^{1} \mathrm{H}$ NMR (300 $\left.\mathrm{MHz}, \mathrm{CDCl}_{3}\right): \delta 4.20(\mathrm{~d}, 1 \mathrm{H}, J=8.9 \mathrm{~Hz}), 5.58(\mathrm{~d}, 1 \mathrm{H}, J=$ $9.2 \mathrm{~Hz}), 6.74(\mathrm{~d}, 2 \mathrm{H}, J=7.6 \mathrm{~Hz}), 6.89(\mathrm{t}, 1 \mathrm{H}, J=7.4 \mathrm{~Hz})$, 7.00 (q, 1H, $J=2.8 \mathrm{~Hz}), 7.21-7.33(\mathrm{~m}, 4 \mathrm{H}) .{ }^{13} \mathrm{C}$ NMR $(75$ $\left.\mathrm{MHz}, \mathrm{CDCl}_{3}\right): \delta 46.1,114.5,117.5,120.6,127.01,127.07$, 127.13, 129.5, 136.8, 144.1. Anal calcd for $\mathrm{C}_{12} \mathrm{H}_{10} \mathrm{~N}_{2} \mathrm{~S}: \mathrm{C}$, 67.26; H, 4.70; N, 13.07; Found: C, 67.30; H, 4.75; N, 12.98 .

2-Phenylamino-hexanenitrile (1j): white solid; recrystallized from ethanol; m.p. $55-57{ }^{\circ} \mathrm{C}\left(56-58{ }^{\circ} \mathrm{C}[10]\right)$. IR $\left(\mathrm{KBr}, \mathrm{cm}^{-1}\right): v 3351(\mathrm{~s}), 2233(\mathrm{~m}) .{ }^{1} \mathrm{H}$ NMR $(300 \mathrm{MHz}$, $\left.\mathrm{CDCl}_{3}\right): \delta 0.85(\mathrm{t}, 3 \mathrm{H}, J=6.7 \mathrm{~Hz}), 1.20-1.40(\mathrm{~m}, 2 \mathrm{H})$, $1.53-1.60(\mathrm{~m}, 2 \mathrm{H}), 3.15(\mathrm{q}, 2 \mathrm{H}, J=6.7 \mathrm{~Hz}), 3.97-4.16(\mathrm{~m}$, $2 \mathrm{H}), 6.98-7.31(\mathrm{~m}, 5 \mathrm{H})$. Anal. Calcd for $\mathrm{C}_{12} \mathrm{H}_{16} \mathrm{~N}_{2}: \mathrm{C}$, 76.55; H, 8.57; N, 14.88; Found: C, 76.51; H, 8.66; N, 14.83 .

2-Benzylamino-3-methyl-butyronitrile (1k): colorless oil [6]. IR (KBr, cm $\left.{ }^{-1}\right): v 3347$ (s), 2223 (m). ${ }^{1} \mathrm{H}$ NMR (300 $\left.\mathrm{MHz}, \mathrm{CDCl}_{3}\right): \delta 1.07(\mathrm{~d}, 3 \mathrm{H}, J=6.7 \mathrm{~Hz}), 1.08(\mathrm{~d}, 3 \mathrm{H}, J=$ $6.8 \mathrm{HZ}), 1.55$ (brs, 1H), 1.96-1.99 (m, 1H), 3.25 (d, 1H, $J=$ $6.0 \mathrm{~Hz}), 3.79(\mathrm{~d}, 1 \mathrm{H}, J=13.0 \mathrm{~Hz}), 4.06(\mathrm{~d}, 1 \mathrm{H}, J=13.0$ $\mathrm{Hz}), 7.23-7.36(\mathrm{~m}, 5 \mathrm{H}) .{ }^{13} \mathrm{C} \mathrm{NMR}\left(75 \mathrm{MHz}, \mathrm{CDCl}_{3}\right): \delta$ $18.2,19.2,31.5,51.7,56.2,119.2,127.4,128.4,128.5$, 138.3. Anal. Calcd for $\mathrm{C}_{12} \mathrm{H}_{16} \mathrm{~N}_{2}$ : C, 76.55; H, 8.57; N, 14.88; Found: C, 76.41; H, 8.48; N, 14.65 .

Morpholine-4-yl-phenyl-acetonitrile (11): white solid; recrystallized from ethanol; m.p. $69-71{ }^{\circ} \mathrm{C}\left(68-69{ }^{\circ} \mathrm{C}\right.$ [6]). IR $\left(\mathrm{KBr}, \mathrm{cm}^{-1}\right): v 2820(\mathrm{~s}), 2230$ (m), 1450 (vs), 1284 (s), 1110 (s), 1002 (s), 915 (s), 862 (s), 738 (s), 684 (s). ${ }^{1} \mathrm{H}$ NMR (300 $\left.\mathrm{MHz}, \mathrm{CDCl}_{3}\right): \delta 2.61(\mathrm{t}, 4 \mathrm{H}, J=4.5 \mathrm{~Hz}), 3.69-3.79(\mathrm{~m}$, $4 \mathrm{H}), 4.85(\mathrm{~s}, 1 \mathrm{H}), 7.37-7.46(\mathrm{~m}, 3 \mathrm{H}), 7.54-7.57(\mathrm{~m}, 2 \mathrm{H})$ ${ }^{13} \mathrm{C}$ NMR $\left(75 \mathrm{MHz}, \mathrm{CDCl}_{3}\right): \delta 50.0,62.46,66.69,115.18$, 128.04, 128.87, 129.12, 132.46. Anal. Calcd for $\mathrm{C}_{12} \mathrm{H}_{14} \mathrm{~N}_{2} \mathrm{O}$ : C, 71.26; H, 6.98; N, 13.85; Found: C, 71.55; H, 7.25; N, 14.01 .

(4-Methoxy-phenyl)-morpholin-4-yl-acetonitrile (1m): white solid; recystalized from ethanol; m.p. $\left.72-74{ }^{\circ} \mathrm{C}\right)$; IR $\left(\mathrm{KBr}, \mathrm{cm}^{-1}\right): v 2925(\mathrm{~s}), 2810(\mathrm{~s}), 2320(\mathrm{~m}), 1610(\mathrm{~m}), 1585$ (m), 1510 (vs), 1465 (s), 1418 (s), 1340 (s), 1320 (s), 1279 (s), 1250 (s), 1235 (vs), 1158 (s), 1140 (s), 1115 (vs), 1020 (s), 1000 (s), 865 (vs), 779 (s). ${ }^{1} \mathrm{H}$ NMR (400 MHz, $\mathrm{CDCl}_{3}$ ): $\delta 2.50-2.58(\mathrm{~m}, 4 \mathrm{H}), 3.68-3.77(\mathrm{~m}, 4 \mathrm{H}), 3.83(\mathrm{~s}, 3 \mathrm{H}), 4.76$ 
(s, 1H), 6.93 (d, 2H, $J=8.8 \mathrm{~Hz}), 7.44(\mathrm{~d}, 2 \mathrm{H}, J=4.8 \mathrm{~Hz})$. ${ }^{13} \mathrm{C} \mathrm{NMR}\left(100 \mathrm{MHz}, \mathrm{CDCl}_{3}\right): \delta 49.88,55.37,61.82,66.68$, $114.14,115.46,124.44,129.30,160.09$. Anal. Calcd for $\mathrm{C}_{13} \mathrm{H}_{16} \mathrm{~N}_{2} \mathrm{O}_{2}$ : C, 67.22; H, 6.94; N, 12.06; Found: C, 66.98; $\mathrm{H}, 6.89 ; \mathrm{N}, 11.92$.

(3,4-Dimethoxy-phenyl)-morpholin-4-yl-acetonitrile (1n): white solid; recystalized from ethanol; m.p. 88-91 $\left.{ }^{\circ} \mathrm{C}\right)$; IR $\left(\mathrm{KBr}, \mathrm{cm}^{-1}\right): v 2925(\mathrm{~s}), 2810(\mathrm{~s}), 2320(\mathrm{~m}), 1610(\mathrm{~m}), 1585$ (m), 1510 (vs), 1465 (s), 1418 (s), 1340 (s), 1320 (s), 1279 (s), 1250 (s), 1235 (vs), 1158 (s), 1140 (s), 1115 (vs), 1020 (s), 1000 (s), 865 (vs), 779 (s). ${ }^{1} \mathrm{H}$ NMR (400 MHz, $\mathrm{CDCl}_{3}$ ): $\delta 2.46-2.55(\mathrm{~m}, 4 \mathrm{H}), 3.60-3.70(\mathrm{~m}, 4 \mathrm{H}), 3.82(\mathrm{~s}, 3 \mathrm{H}), 3.84$ (s, 3H), $4.69(\mathrm{~s}, 1 \mathrm{H}), 6.80(\mathrm{~d}, 1 \mathrm{H}, J=8.3 \mathrm{~Hz}), 6.94(\mathrm{~d}, 1 \mathrm{H}, J$ $=2.0 \mathrm{~Hz}), 7.03\left(\mathrm{dd}, 1 \mathrm{H}, J_{1}=8.0 \mathrm{~Hz}, J_{2}=2.0 \mathrm{~Hz}\right) .{ }^{13} \mathrm{C} \mathrm{NMR}$ $\left(100 \mathrm{MHz}, \mathrm{CDCl}_{3}\right): \delta 49.91,55.97,56.02,62.10,66.69$, $110.72,110.83,115.40,120.47,124.81,149.28,149.56$. Anal. Calcd for $\mathrm{C}_{14} \mathrm{H}_{18} \mathrm{~N}_{2} \mathrm{O}_{3}$ : C, 64.10; H, 6.92; N, 10.68; Found: C, 63.89; H, 6.88; N, 10.51 .

\section{Results and discussion}

SBPDSA was prepared by the reaction of DTPS and chlorosulfonic acid in chloroform. The BET surface area calculated from nitrogen adsorption isotherms measured at the temperature of liquid nitrogen was $762 \mathrm{~m}^{2} / \mathrm{g}$ and the total pore volume was calculated to be $0.1342 \mathrm{~cm}^{3} / \mathrm{g}$. The content of $\mathrm{S}$ obtained from elemental analysis showed that typically a loading of $0.99 \mathrm{mmol} / \mathrm{g} \mathrm{H}^{+}$was obtained. Thermal stability of a catalyst is an important requirement. The TG curve for the catalyst is shown in Fig. 1. Mass loss began at about $120^{\circ} \mathrm{C}$, which indicated decomposition of the catalyst. Therefore, the covalent bonds in the catalyst endow it with high thermal stability.

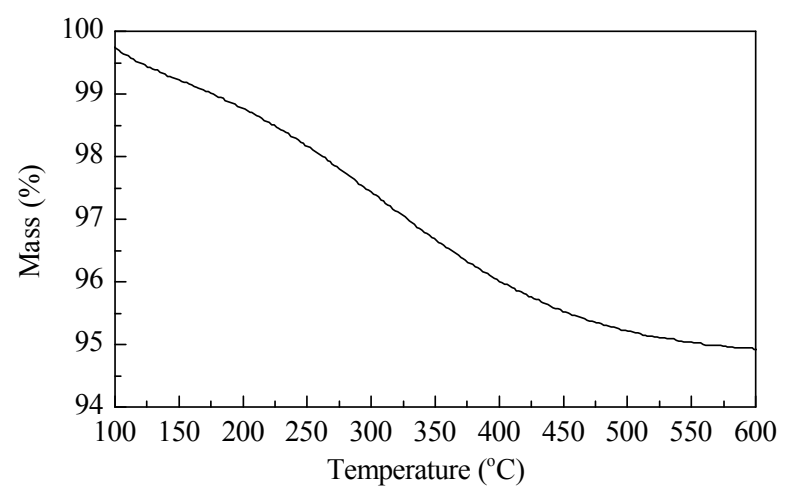

Fig. 1. TG curve of SBPDSA.

To study the effect of catalyst loading on the condensation reaction of aldehydes and amines with TMSCN into the corresponding $\alpha$-aminonitriles, the reaction of benzaldehyde and aniline with TMSCN was chosen as a model reaction (Table 1). It can be seen that the reaction did not proceed even after $24 \mathrm{~h}$ in the absence of this catalyst (Table 1, entry 1). Obviously, SBPDSA is important to catalyze the reaction. The optimal amount of SBPDSA was found to be 0.07 $\mathrm{g}(6.9 \mathrm{~mol} \%)$ per $1 \mathrm{mmol}$ of aldehyde in ethanol at room temperature. Although a lower catalyst loading of 0.05 or $0.03 \mathrm{~g}$ accomplished this condensation, $0.07 \mathrm{~g}$ of SBPDSA per $1 \mathrm{mmol}$ of aldehyde was optimal in terms of reaction time and isolated yield.

Table 1 Condensation of benzaldehyde, aniline, and TMSCN in the presence of different amounts of SBPDSA

\begin{tabular}{cccc}
\hline Entry & Catalyst loading $(\mathrm{g} / \mathrm{mol} \%)$ & Time $(\mathrm{min})$ & Yield $^{*}(\%)$ \\
\hline 1 & - & 24 & $<10$ \\
2 & $0.03 / 2.9$ & 140 & 73 \\
3 & $0.05 / 4.9$ & 110 & 78 \\
4 & $0.07 / 6.9$ & 5 & 93 \\
5 & $0.09 / 8.9$ & 5 & 92 \\
\hline
\end{tabular}

Reaction conditions; benzaldehyde $1 \mathrm{mmol}$, aniline $1.5 \mathrm{mmol}$, TMSCN $1.5 \mathrm{mmol}$, EtOH $2 \mathrm{ml}$, room temperature. "Isolated yield.

We used the optimized conditions $(0.07 \mathrm{~g} / \mathrm{mmol}$ of SBPDSA in ethanol at room temperature) for the condensation reaction of various aryl aldehydes and amines with TMSCN into the corresponding $\alpha$-aminonitriles. As shown in Table 2, both aromatic and aliphatic aldehydes reacted with amines and TMSCN in the presence of SBPDSA in this one-pot condensation to afford the corresponding $\alpha$-aminonitriles in excellent yield. Moreover, aldehydes with electron-donating or electron-withdrawing groups, i.e., 4-methoxybenzaldehyde, 3,4,5-trimethoxybenzaldehyde, and 3-nitrobenzaldehyde, were converted into the corresponding $\alpha$-aminonitriles $\mathbf{1 e - 1 h}$ in high yield (Table 2, entries 5-8). The acid sensitive substrate thiophene-2-carbaldehyde gave the expected $\alpha$-aminonitrile $\mathbf{1 i}$ in very good yield (Table 2, entry 9). Aliphatic aldehydes such as pentanal and 2-methylpropanal gave the corresponding products $\mathbf{1 j}$ and $\mathbf{1 k}$ in $73 \%$ and $75 \%$ yield, respectively (Table 2, entries 10 and 11). Also, as shown in Table 2, primary amines such as aniline, 4-chloroaniline, and benzylamine, and secondary amines such as morpholine were suitable for use in this one-pot condensation reaction. The reactions progressed rapidly at room temperature and gave very good yields.

The possibility of recycling the catalyst was examined using the reaction of benzaldehyde and aniline with TMSCN under the optimized conditions. Upon completion of the reaction, the mixture was filtered and then washed with warm ethanol. The product was recrystallized from hot ethanol. The recovered catalyst was dried and reused for subsequent runs. The recycled catalyst could be reused five times without any additional treatment or appreciable reduction in catalytic activity (Fig. 2). 
Table 2 Preparation of various $\alpha$-aminonitriles in the presence of SBPDSA in EtOH at room temperature

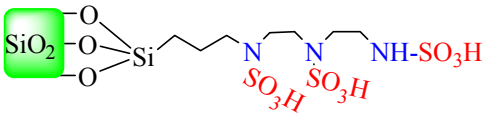

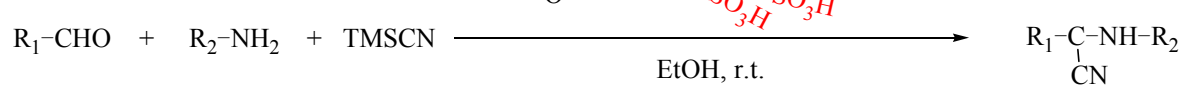

\begin{tabular}{|c|c|c|c|c|c|c|c|}
\hline \multirow{3}{*}{ Entry } & \multirow{3}{*}{$\mathrm{R}_{1}$} & \multirow{3}{*}{$\mathrm{R}_{2}$} & \multirow{3}{*}{ Product } & \multirow{3}{*}{$\begin{array}{l}\text { Time } \\
(\min )\end{array}$} & \multicolumn{3}{|c|}{$1 a-1 n$} \\
\hline & & & & & Yield $^{*}$ & \multicolumn{2}{|c|}{ Melting point $\left({ }^{\circ} \mathrm{C}\right)$} \\
\hline & & & & & $(\%)$ & Found & Reported \\
\hline 1 & $\mathrm{C}_{6} \mathrm{H}_{5}-$ & $\mathrm{C}_{6} \mathrm{H}_{5}-$ & 1a & 5 & 93 & $80-82$ & $81-83[12]$ \\
\hline 2 & 4- $\mathrm{ClC}_{6} \mathrm{H}_{4-}$ & $\mathrm{C}_{6} \mathrm{H}_{5-}$ & $1 \mathrm{~b}$ & 5 & 85 & $109-111$ & $111-112[8]$ \\
\hline 3 & 4- $\mathrm{BrC}_{6} \mathrm{H}_{4-}-$ & $\mathrm{C}_{6} \mathrm{H}_{5-}$ & $1 \mathrm{c}$ & 5 & 87 & $101-103$ & $99-100[5]$ \\
\hline 4 & $2,4-(\mathrm{Cl})_{2} \mathrm{C}_{6} \mathrm{H}_{3}-$ & $\mathrm{C}_{6} \mathrm{H}_{5-}$ & 1d & 4 & 91 & $115-117$ & $115-117[14]$ \\
\hline 5 & 4- $\mathrm{CH}_{3} \mathrm{OC}_{6} \mathrm{H}_{4-}$ & $\mathrm{C}_{6} \mathrm{H}_{5-}$ & $1 \mathrm{e}$ & 5 & 93 & $95-97$ & $94-95[8]$ \\
\hline 6 & $3,4,5-(\mathrm{MeO}){ }_{3} \mathrm{C}_{6} \mathrm{H}_{2}-$ & $\mathrm{C}_{6} \mathrm{H}_{5-}$ & 1f & 5 & 90 & $147-149$ & $147-149[14]$ \\
\hline 7 & $3,4,5-(\mathrm{MeO}){ }_{3} \mathrm{C}_{6} \mathrm{H}_{2}-$ & 4-Cl- $\mathrm{C}_{6} \mathrm{H}_{4-}$ & $1 \mathrm{~g}$ & 5 & 90 & $145-148$ & - \\
\hline 8 & $3-\mathrm{O}_{2} \mathrm{NC}_{6} \mathrm{H}_{4-}$ & $\mathrm{C}_{6} \mathrm{H}_{5-}$ & $1 \mathrm{~h}$ & 20 & 92 & $88-91$ & $89-92[14]$ \\
\hline 9 & 2-Thionyl- & $\mathrm{C}_{6} \mathrm{H}_{5-}$ & $1 \mathbf{i}$ & 50 & 78 & $97-99$ & $98-100[11]$ \\
\hline 10 & $\mathrm{CH}_{3}\left(\mathrm{CH}_{2}\right)_{2} \mathrm{CH}_{2-}$ & $\mathrm{C}_{6} \mathrm{H}_{5-}$ & $\mathbf{1 j}$ & 5 & 73 & $55-57$ & $56-58[10]$ \\
\hline 11 & $\left(\mathrm{CH}_{3}\right)_{2} \mathrm{CH}-$ & $\mathrm{C}_{6} \mathrm{H}_{5}-\mathrm{CH}_{2}-$ & $1 \mathrm{k}$ & 5 & 75 & colorless oil & colorless oil [6] \\
\hline 12 & $\mathrm{C}_{6} \mathrm{H}_{5-}$ & $\mathrm{HN} \quad \mathrm{O}$ & 11 & 5 & 91 & 69-71 & $68-69[6]$ \\
\hline 13 & $4-\mathrm{MeOC}_{6} \mathrm{H}_{4-}$ & $\mathrm{HN}$ & $1 \mathrm{~m}$ & 5 & 89 & $72-74$ & - \\
\hline 14 & $3,4-(\mathrm{MeO})_{2} \mathrm{C}_{6} \mathrm{H}_{3}-$ & $\mathrm{HN}$ & 1n & 5 & 90 & $88-91$ & - \\
\hline
\end{tabular}

Reaction conditions: aldehyde $1 \mathrm{mmol}$, amine $1.5 \mathrm{mmol}$, TMSCN $1.5 \mathrm{mmol}$, SBPDSA $0.07 \mathrm{~g}$, EtOH $2 \mathrm{ml}$. *Isolated yield.

Table 3 Comparison of different solid acid catalysts for the condensation reaction of benzaldehyde, aniline, and TMSCN

\begin{tabular}{|c|c|c|c|c|}
\hline Catalyst & Catalyst loading $(\mathrm{g} / \mathrm{mmol})$ & Time (min) & Yield $^{\mathrm{a}}(\%)$ & Ref. \\
\hline Montmorillonite KSF clay & 1.0 & 210 & 90 & [8] \\
\hline Silica sulfuric acid & $0.095 / 0.25$ & 360 & 88 & [9] \\
\hline Xanthan sulfuric acid & $0.1 / 0.06$ & 65 & 97 & [12] \\
\hline SBA-15 supported sulfonic acid & $0.031 / 0.017$ & 5 & $100^{\mathrm{b}}$ & [13] \\
\hline SBSSA & $0.2 / 0.066$ & 30 & 94 & [14] \\
\hline Sulfamic acid $\mathrm{Fe}_{3} \mathrm{O}_{4}$ nanoparticles & $0.02 / 0.006$ & 10 & 97 & [15] \\
\hline SBPDSA & $0.07 / 0.069$ & 5 & 93 & this work \\
\hline
\end{tabular}

${ }^{\text {a Issolated yield. }}{ }^{\mathrm{b}}$ The reaction was performed at $50^{\circ} \mathrm{C}$.

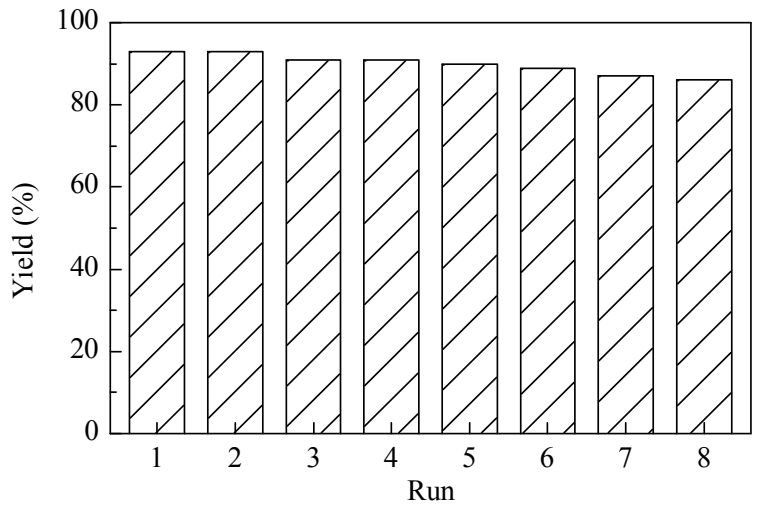

Fig. 2. Recyclability of SBPDSA as a catalyst in the reaction of benzaldehyde and aniline with TMSCN. Reaction conditions: benzaldehyde $1 \mathrm{mmol}$, aniline $1.5 \mathrm{mmol}$, TMSCN $1.5 \mathrm{mmol}$, at room temperature, $5 \mathrm{~min}$.
Finally, a comparative study of SBPDSA with other recently reported catalysts for condensation of benzaldehyde and aniline with TMSCN was made, which revealed that SBPDSA is equally as efficient and reusable as other catalysts (Table 3).

\section{Conclusions}

An efficient method was developed for the synthesis of $\alpha$-aminonitriles in high yield by using SBPDSA as a catalyst at room temperature. The catalyst was recovered and reused without any noticeable loss of activity. The heterogeneous conditions, green solvent, easy and clean work up, and simplicity of this procedure are improvements over many existing methods. 


\section{References}

1 Strecker A. Ann Chem Pharm, 1850, 75: 27

2 March J. Advanced Organic Chemistry. 4th Ed. New York: Wiley, 1995. 965

3 Weinstock L M, Davis P, Handelsman B, Tull R. J Org Chem, 1967, 32: 2823

4 Matier W L, Owens D A, Comer W T, Dietchman D, Ferguson H C, Seidehamel R J, Young J R. J Med Chem, 1973, 16: 901

5 El-Ahl A A S. Synth Commun, 2003, 33: 989

6 Ranu B C, Dey S S, Hajra A. Tetrahedron, 2002, 58: 2529

7 Yadav J S, Reddy B V S, Eshwaraiah B, Srinivas M, Vishnumurthy P. New J Chem, 2003, 27: 462

8 Yadav J S, Reddy B V S, Eswaraiah B, Srinivas M. Tetrahedron, 2004, 60: 1767

9 Chen W Y, Lu J. Synlett, 2005: 2293

10 Wang H S, Zhao L F, Du Z M. Chin J Chem, 2006, 24: 135

11 Khan N H, Agrawal S, Kureshy R I, Abdi S H R, Singh S, Suresh E, Jasra R V. Tetrahedron Lett, 2008, 49: 640

12 Shaabani A, Maleki A, Soudi M R, Mofakham H. Catal Commun, 2009, 10: 945

13 Karimi B, Zareyee D. J Mater Chem, 2009, 19: 8665

14 Niknam K, Saberi D, Nouri Sefat M. Tetrahedron Lett, 2010, 51: 2959

15 Kassaee M Z, Masrouri H, Movahedi F. Appl Catal A, 2011, 395: 28
16 Nouri Sefat M, Saberi D, Niknam K. Catal Lett, 2011, 141: 1713

17 Leblanc J-P, Gibson H W. Tetrahedron Lett, 1992, 33: 6295

18 Heydari A, Fatemi P, Alizadeh A A. Tetrahedron Lett, 1998, 39: 3049

19 Das B, Ramu R, Ravikanth B, Reddy K R. Synthesis, 2006 : 1419

20 Karimi B, Zareyee D. Org Lett, 2008, 10: 3989

21 Niknam K, Zolfigol M A, Hossieninejad Z, Daneshvar N. Chin J Catal, 2007, 28: 591

22 Niknam K, Saberi D. Appl Catal A, 2009, 366: 220

23 Niknam K, Saberi D, Nouri Sefat M. Tetrahedron Lett, 2009, 50: 4058

24 Niknam K, Saberi D. Tetrahedron Lett, 2009, 50: 5210

25 Niknam K, Panahi F, Saberi D, Mohagheghnejad M. J Heterocycl Chem, 2010, 47: 292

26 Niknam K, Saberi D, Molaee H, Zolfigol M A. Can J Chem, 2010, 88: 164

27 Niknam K, Mohammadizadeh M R, Mirzaee S, Saberi D. Chin J Chem, 2010, 28: 663

28 Tayebi S, Baghernejad M, Saberi D, Niknam K. Chin J Catal, 2011, 32: 1477

29 Niknam K, Mohammadizadeh M R, Mirzaee S. Chin J Chem, 2011, 29: 1417

30 Nouri Sefat M, Deris A, Niknam K. Chin J Chem, 2011, 29: 2361 